\title{
Improving timeliness of newborn screens in the neonatal intensive care unit: a quality improvement initiative
}

\author{
Kelechi Ikeri $\mathbb{D}^{1} \cdot$ Vilmaris Quinones Cardona $\rrbracket^{1,2} \cdot$ Ogechukwu R. Menkiti $\circledast^{1,2}$
}

Received: 21 October 2020 / Revised: 14 January 2021 / Accepted: 3 February 2021 / Published online: 23 March 2021

(c) The Author(s), under exclusive licence to Springer Nature America, Inc. 2021

\begin{abstract}
Background Despite the established utility of newborn screening tests (NBS), achieving timely specimen transit is a challenge for neonatal intensive care units (NICU).

Methods This project was conducted between September 2017 and July 2020 using the Plan-Do-Study-Act (PDSA) tool. Our primary aim was to increase the percent of NBS samples reaching the state laboratory within 1 day of collection by $20 \%$ by April 2020. Process, outcome, and balancing measures were monitored.

Results Five hundred and eighty-five NBS were collected. There was special cause variation with improvement in the percent of samples received within 1 day of collection from 28 to $77 \%$. Special cause variation was also observed in the process measures without an increase in the percent of unacceptable samples.

Conclusions Standardizing the NBS collection processes by adopting a sample collection window and same day courier pickup ensures timely specimen transit without adversely affecting the quality of samples collected.
\end{abstract}

\section{Introduction}

The newborn screen (NBS) program is one of the largest public health initiatives involving newborns in the United States. Since the development of screening tests for phenylketonuria by Robert Guthrie in the 1960s, the number of diseases detected by this program has expanded. Currently, testing is available for more than 50 disorders in most states and to be effective, all involved processes and steps must be efficient and timely [1].

Traditionally, the NBS process has been divided into three phases. The time from birth to NBS sample arrival at the testing laboratory is termed the pre-analytic phase. The analytic phase includes all events in the laboratory through generation of results. The post-analytic phase encompasses all the processes from reporting and receipt of results to the

Supplementary information The online version contains supplementary material available at https://doi.org/10.1038/s41372021-00985-z.

Vilmaris Quinones Cardona

Vq23@drexel.edu

1 St. Christopher's Hospital for Children, Philadelphia, PA, USA

2 Drexel University College of Medicine, Philadelphia, PA, USA completion of the follow up actions and closure of each case [2].

Timeliness of the NBS process is important to reduce morbidity and mortality. Time-critical metabolic conditions like amino acid disorders require immediate treatment, with hours of delay resulting in increased risk of mortality [3]. Diagnostic tests for most time-critical conditions have long turnaround times (TATs) hence the NBS serves as an important early screening tool.

The NewSTEPS 360 program was developed in 2015 to support state NBS programs in an effort to improve timeliness. This initiative, funded by the Health Resources and Services Administration, has improved various quality benchmarks among state participants [2].

Current NBS timeliness goals established by the Advisory Committee on Heritable Disorders in Newborn and Children (ACHDNC) in April 2015 are recognized nationally as quality indicators $[2,3]$. The sole purpose of these goals is to ensure timely screening in the presymptomatic phase thereby reducing disability, morbidity, and mortality. These benchmarks are standards for specimen collection, transit and result reporting. For specimen transit, the specific goal is for all samples to reach the state laboratory ideally within one calendar day [2]. Despite improvements in overall timeliness, few NewSTEPs 360 participants have met the ACHDNC target of timely transit of specimens to 
state laboratories. In the United States between 2016 and 2018 , only $39-42 \%$ of specimens from NBS programs in 25 participating states were received within one calendar day despite the implementation of suggested interventions [2].

For nonparticipating centers like ours, achieving timeliness goals also remain a challenge.

During the baseline period (September 2017-April 2019), only $34 \%$ of the samples were same day courier pickups with $28 \%$ arriving at the state laboratory within one calendar day. Also, $76 \%$ of filter papers were sent with missing information. These delays could result in devastating consequences such as in cases of congenital hypothyroidism where delayed treatment could lead to poor neurodevelopmental outcomes [4, 5]. In our unit, two infants with presumptive alpha-L-iduronidase deficiency had results reported at 18 and 22 days after sample collection causing delays in initiating appropriate management.

\section{Specific aims}

Our SMART (Specific, Measurable, Achievable, Realistic, and Timely) AIM was to increase the percent of samples reaching the state laboratory within 1 calendar day of collection by $20 \%$ (from 28 to $48 \%$ ) by April 2020 .

\section{Methods}

\section{Context}

This quality improvement project was conducted between September 2017 and July 2020 in the neonatal intensive care unit (NICU) at St. Christopher's Hospital for Children using the Model for Improvement's Plan-Do-Study-Act (PDSA) tool to gather knowledge, test and implement interventions. This unit is a 39-bed level IV out-born tertiary referral center. Clinical providers include neonatology attending physicians, neonatology fellows, nurse practitioners, physician assistants, nurses and rotating pediatric residents. All patients admitted to the NICU are transferred from other hospitals and initial samples are customarily obtained prior to transfer. State regulations stipulate that samples should not be collected before $24 \mathrm{~h}$ of life because of higher false negative rates and increased potential to miss life-threatening metabolic conditions [3]. Most of our NBS samples are repeat specimens.

The unit utilizes an electronic health record (EHR) system and the NBS can be checked as part of a power plan or ordered separately. After EHR order entry by the provider, the NBS sample is collected by the patient's nurse who hands it to the unit secretary. Safe keeping of NBS filter papers, pre- and post-sample collection, processing, and subsequent dispatch to the hospital laboratory is ensured by the unit secretary. The hospital has a central laboratory that processes the NBS samples after receipt from the NICU and a unique tracking number is generated by laboratory personnel prior to courier shipping. Samples are shipped to the state laboratory on 6 calendar days per week excluding Sundays. Specimens drawn on Sundays are stored in the hospital laboratory until courier pickup the following day. All samples in Pennsylvania are subsequently processed at the PerkinElmer laboratory in Pittsburgh, PA. Data on individual specimen TAT, percent of unacceptable samples, and percent of samples with missing information are provided to the NICU Medical Director by the state laboratory on a monthly basis.

Process mapping identified barriers and process redundancies (Supplementary Fig. 1). Feedback from key stakeholders in the NBS program shed light on the common causes of delay: erratic timing of sample collection, inconsistent collection techniques, lack of standardized sample processing, and inconsistent pickup by courier services. A driver diagram was created to identify primary drivers and formulate interventions required to reduce delays (Fig. 1).

\section{Interventions}

We assembled a quality improvement team comprising two neonatology attending physicians, a neonatology fellow as well as a pediatric resident, nursing, clerical, and laboratory staff champions. Interventions were targeted at problems identified in the various phases of the NBS process and implemented through a series of PDSA cycles. Problems with timing of specimen collection were addressed through educational sessions, use of the NBS algorithm, the NBS calendar and implementation of a sample collection window. Nursing in-service, the use of visual reminders and review of filter papers by the nursing champion were aimed at standardizing sample collection techniques. The placard system was introduced to tackle inconsistencies with courier pickup.

\section{Educational sessions}

These sessions were conducted to prepare providers for upcoming changes to the NBS process and to fill gaps in knowledge of appropriate NBS sampling times based on the current Pennsylvania guidelines. Informal provider education began in April 2019 and a formal educational series was started in June 2019 during the weekly NICU conference. Brief sessions were subsequently conducted monthly for pediatric residents on the first day of their NICU rotation.

\section{NBS algorithm, calendar, and handoff}

Despite ongoing educational interventions, providers experienced difficulties in identifying and appropriately timing 


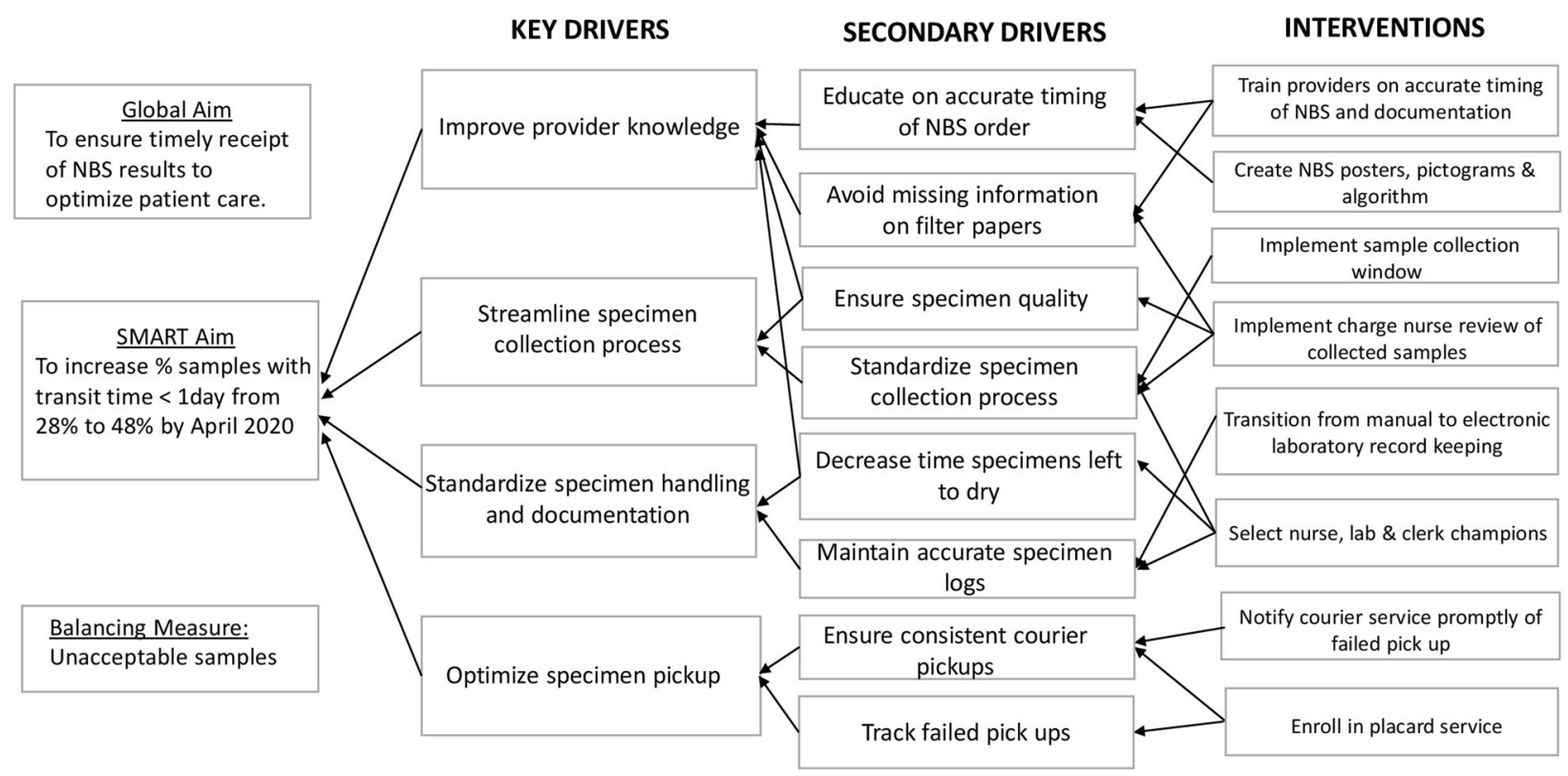

Fig. 1 Key Driver Diagram summarizing the project aim, drivers and interventions required to achieve smart aim.

repeat NBS tests. A NBS admission algorithm was designed to help providers navigate these challenging situations. Educational presentations were revised to include this algorithm.

The NBS calendar and documentation in handoff sheets were introduced to facilitate appropriate timing of repeat samples. Providers were required to manually enter the patient's name below the scheduled test date on the calendar. This information was shared with nursing and clerical champions to facilitate timely filter paper preparation. Providers were also asked to document the dates of upcoming NBS in the patient handoff.

\section{Sample collection window}

This intervention was implemented to address erratic timing of specimen collection. Review of baseline data provided by the state laboratory revealed a higher percent of samples with transit time less than one calendar day in February 2019. Most of these samples were collected between 3 a.m. and $11 \mathrm{a}$ a.m. and preferentially timed by the physician team to coincide with other morning laboratory samples to minimize additional heel sticks. Further study showed that morning sample collection also allowed for adequate sample preparation and processing before the stipulated 4 p.m. courier pickup. We hypothesized that modification of sample collection time to a morning window would improve transit time by eliminating redundancies in handling prior to shipping. Through implementation of PDSA ramp to drive improvement (Fig. 2), a morning collection time was implemented in May 2019. This intervention was modified in June 2019 to include a narrower collection window from 8 to 10 a.m. in routine situations so that samples were collected and processed in the same shift to minimize handoffs. This collection window was required to fall within $24-48 \mathrm{~h}$ of life, with the exception of repeat samples collected beyond $48 \mathrm{~h}$ of life. However, providers were reminded to obtain NBS samples prior to emergency transfusion of packed red blood cells, even if the patient was $<24 \mathrm{~h}$ old and the timing of collection fell outside of the window. In these instances, repeat samples were obtained following state guidelines.

\section{Nursing in-service and visual reminders}

To standardize sample collection techniques, nursing staff in-service on sample collection and processing were organized by the nursing champions. NBS posters and pictograms were created and placed in strategic NICU areas to serve as visual reminders and to provide information on proper sampling methods [6].

\section{Nursing champion review of filter papers}

To address the high percent of samples sent to the state laboratory with missing information, starting in June 2019, filter paper samples were required to be reviewed by the available nursing champion or unit secretary. This served as quality assurance prior to processing and dispatch to the hospital laboratory.

\section{Placard service}

Local laboratory records showed inconsistent sample pickups by the courier. A placard service was requested in June 
Fig. 2 PDSA testing ramp for improvement illustrating the PDSA cycles implemented to achieve SMART Aim.

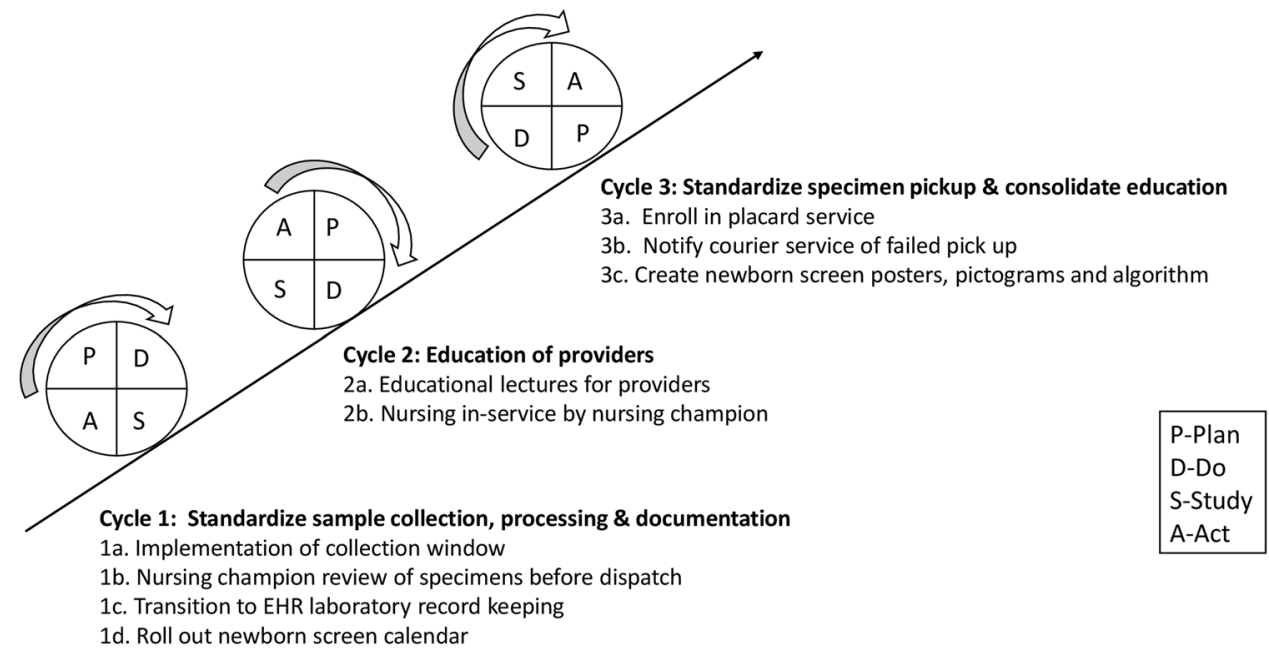

2019 and successfully implemented in September 2019 at no extra cost. It involved placement of a provided barcoded plaque on the NBS courier collection box and courier personnel scanning at sample pickup time. This allowed for real time team notification of successful pickup. Failed pickups were promptly identified and immediately rescheduled online by the fellow physician team member. Information on failed pickups was relayed to the courier company contact to aid in root cause analysis, avoid recurrences and foster resolution.

\section{Measures}

Process measures included the percent of samples obtained within the instituted collection window and the percent of dried blood spot filter papers sent with missing information or incompletely filled. Data entry and documentation compliance was monitored through review of patients' EHR, handoff sheets, the NBS calendar and laboratory records. We also tracked the percent of samples picked up on the same day by the courier service and the monthly percent of NBS samples reaching the state laboratory within 1 day of collection. To determine the impact of our interventions on the entire NBS process, we monitored the percent of samples with TAT $<3$ days. TAT was defined as time from sample collection to result reporting. Specimen transit time and TAT data on each sample was provided to a designated physician monthly by the state laboratory.

To further highlight clinical relevance, we compared the percent of patients with presumptive diagnosis made $<3,4$, and 5 calendar days after sample collection pre- and postintervention as an outcome measure.

We recognized that the implementation of a strict sample collection window could inadvertently provoke undue stress among nursing staff potentially affecting the quality of specimens obtained. For this reason, the percentage of unacceptable samples was chosen as a balancing measure. Unacceptable samples were defined by the laboratory as: quantity not sufficient for testing, oversaturated, diluted, discolored, clotted, double spotted or contaminated specimens, specimens not soaked through to the back of filter paper, and specimens mailed while blood is wet or got wet in transit [6].

\section{Study of interventions}

Process and balancing measures were represented using annotated P statistical process control (SPC) charts. The outcome measure was represented on a bar graph comparing pre- and post-intervention data. QI-Macros 2019 was used to analyze and generate SPC charts. Centerlines and 3$\sigma$ control limits were defined using standard approaches. Special cause variation was detected and established rules were applied when at least eight consecutive points were above or below the center line, one or more data points fell beyond the control limit or six consecutive points trended in either direction [7,8]. Centerlines were adjusted based on detection of special cause signal [9]. This initiative was reviewed by the Drexel University Institutional Review Board and determined to not meet the definition of human subject research.

\section{Results}

A total of 585 NBS samples were collected: 361 samples during the 20-month baseline period from September 2017 to April 2019, and 224 samples during the intervention period between May 2019 and July 2020. All NBS samples were included and there were no missing or lost samples. There was special cause variation in the percent of samples collected between 8 and 10 a.m. from a baseline of 4.6 to 



Fig. 3 P charts. A Specimens collected within the designated window. B Filter papers with missing information. C Same day pickups. D Specimen with transit time $<1$ day. E Specimen turnaround times $<3$ days.

$78 \%$ after implementation of the sample collection window (Fig. 3A). Monthly fluctuations in this measure reflected either noncompliance or higher volume of acute cases with emergent need for blood transfusion. There was special cause variation and a center-line shift in filter papers missing information with a decrease from 76.2 to $17 \%$ (Fig. 3B). Provider documentation compliance was $92.2 \%$ with the admission note, $83.1 \%$ with the handoff sheets, and $66.4 \%$ with the calendar after interventions.

For same day courier pickup, initial review showed special cause variation in the baseline data with one data point (February 2019) falling above the upper control limit. Further study showed that most NBS samples obtained that month were timed with collection of other morning samples at the preference of the on-service physician. This data point was not incorporated into the calculation of the center line or control limits. With interventions, special cause variation was observed in same day pickup with improvement from 33.9 to $93 \%$ (Fig. 3C). After sequential process changes, there was special cause variation with center-line shift in the percent of samples reaching the state laboratory within 1 day from 28 to $77 \%$, exceeding our goal of $48 \%$ (Fig. 3D). Special cause variation was also observed in the percent of samples with TAT $<3$ days with improvement from 39.1 to $70 \%$ (Fig. 3E).

Compared to baseline, a higher percent of patients were presumptively diagnosed at $<3,4$, and 5 days of sample collection respectively following interventions (Fig. 4). 
Fig. 4 Time to presumptive diagnosis. Percent of patients who were presumptively diagnosed in $<3,4$ and 5 days from NBS collection pre and post-intervention.
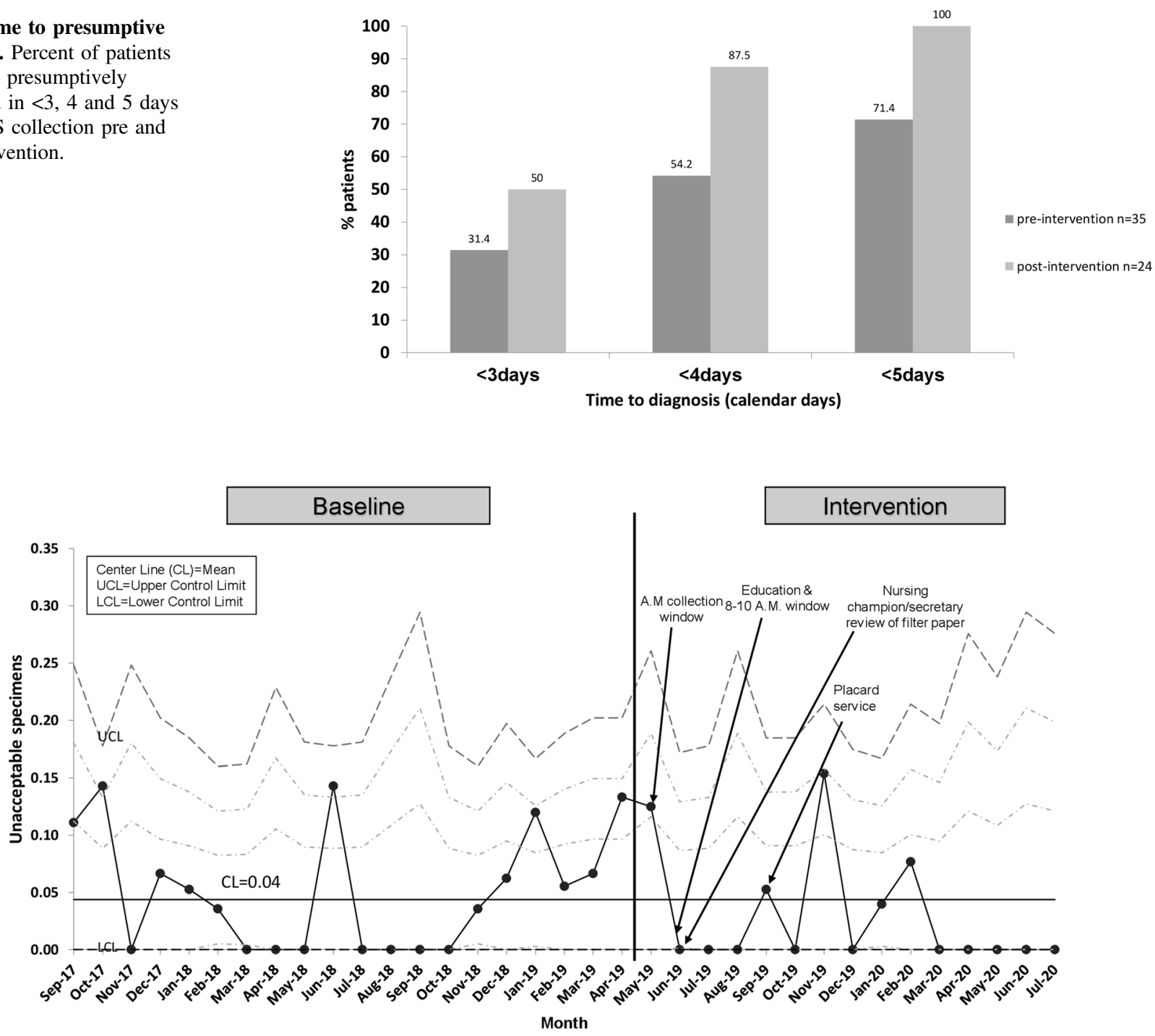

Fig. 5 Balancing measure $\mathbf{P}$ chart. Percent of patients with unacceptable NBS specimens.

Regarding the balancing measure, the percent of unacceptable specimens remained minimal with a mean of $4 \%$ (Fig. 5).

\section{Discussion}

Timeliness of the NBS system facilitates early diagnosis of critical and noncritical medical conditions allowing for prompt initiation of management strategies. Preventing NBS process delays is critical to achieving timeliness goals. ACHDNC timeliness goals apply to initial specimens collected and are reported in relation to time of birth [2]. For Level IV out-born NICUs where most NBS samples collected are repeat specimens, computing timeliness data in reference to the time of collection is more reflective of the NBS process.
Achieving timeliness of specimen transit remains a challenge even for NewSTEPs 360 participating centers. Sontag et al. reported only $41 \%$ of samples arrived at the laboratory within 1 calendar day [2]. A recent study assessing the Michigan NBS program also identified the time between specimen collection and receipt by the state laboratory as a significant bottleneck in the process. The authors concluded that modifying sample pickup time to 9 p.m. could reduce specimen transit delays [10]. In our initiative, we demonstrated that improved specimen transit time is attainable with a pickup as early as 4 p.m. without comprising specimen quality. Afternoon pickup allowed for same day morning sample collection and ample time for processing $(3 \mathrm{~h}$ drying time and in-house laboratory processing). To achieve same day pickup, a strategic modification to a morning sample collection window was essential. A challenge we faced in the adherence to this 
collection window was the need for emergency transfusions among high acuity patients altering the timing of specimen collection. We also encountered courier service interruptions imposed by the COVID-19 pandemic leading to a transient decrease in same day pickup in April 2020. Despite these challenges, the overall timeliness was not adversely affected likely due to the cumulative effect of other implemented interventions. The improvement in the percent of samples with transit time $<1$ calendar day to $77 \%$ is particularly important for time-critical metabolic conditions. As a result of pre-analytic improvement in timeliness, a higher percent of presumptive positive cases was promptly detected allowing for earlier subspecialty referrals and treatment.

Samples sent with missing information also cause delays in the NBS process. Strategies adopted by NewSTEPs 360 programs to tackle this problem focused solely on education of providers [2]. In comparison, we demonstrated a significant reduction in the percent of samples with missing information through education in addition to the implementation of filter paper review prior to dispatch.

Our balancing measure, as defined by the percent of unacceptable samples, did not increase with our interventions.

\section{Limitations}

This quality improvement project was conducted in a Level IV NICU with specialized resources, a dedicated in-house laboratory and established courier service which may limit its generalizability. However, creative interventions such as a establishing a sample collection window and optimization of courier services can be more readily incorporated in the workflow of all level NICUs with invested leadership and staff.

Our work focused on the pre-analytic phase of the NBS process. We recognize that problems in any of the three phases of NBS processing can lead to delayed result reporting despite local efforts to improve timeliness. However, we demonstrated that directed efforts to the preanalytic phase improved overall timeliness and further work is necessary in the other steps to further impact our TAT. In terms of the data itself, we had no means of assessing the degree to which each identified factor contributed to TAT delays. This information would have been useful for determining priority of interventions.

\section{Conclusion}

This quality initiative serves as a roadmap for improving NBS timeliness in the NICU and demonstrates success through implementation of strategic interventions addressing barriers to timely specimen transit. More importantly, this initiative highlights earlier detection of presumptive positive cases is attainable without adversely affecting the quality of samples collected or comprehensiveness of information required for processing in a Level IV NICU. Continued surveillance is crucial for long-term sustainability, hence appointed champions will continue to drive project aims and the sample collection window will be incorporated into local clinical practice guidelines. Future directions include expanding these efforts to the local outpatient pediatric and special needs clinic, modification of sample collection window to ensure synchrony with clinic workflow and training of clinic staff on specimen collection.

Further work is needed to understand the role of the analytic and post-analytic phases in overall timeliness.

Acknowledgements The authors acknowledge the tremendous efforts of our key champions Jillian Taylor MD, Sharon Leonardo RN, Leann Mason RN, Milagros Pina, Jeanette Rodriguez, Terrilyn Rynkiewicz, Nikki Beckel, and Rebecca Bausinger. We also want to thank the entire NICU team who made implementation and sustainability of this initiative possible.

Author contributions KI conceptualized and designed the study, collected data, participated in the analysis, and drafted the initial paper. VQC conceptualized and designed the study, assisted and supervised data collection, carried out analysis, reviewed the data, critically reviewed, and revised the paper for important intellectual content. ORM conceptualized and designed the study, assisted in data collection, critically reviewed, and revised the paper for intellectual content. All authors approved the final paper as submitted and agree to be accountable for all aspects of the work.

\section{Compliance with ethical standards}

Conflict of interest The authors declare no competing interests.

Publisher's note Springer Nature remains neutral with regard to jurisdictional claims in published maps and institutional affiliations.

\section{References}

1. Kronn D. Navigating newborn screening in the NICU: a user's guide. Neoreviews. 2019;20:e280-91.

2. Sontag MK, Miller JI, Mckasson S, Sheller R, Edelman S, Yusuf C, et al. (2020) Newborn screening timeliness quality improvement initiative: impact of national recommendations and data repository. PLoS ONE. 2020;15:e0231050.

3. Newborn Screening Timeliness Goals. Health resources and service administration. 2015. https://www.hrsa.gov/advisory-committees/ heritable-disorders/newborn-screening-timeliness.

4. Wassner AJ. Congenital hypothyroidism. Clin Perinatol. 2018;45: $1-18$.

5. Weiner A, Oberfield S, Vuguin P. The laboratory features of congenital hypothyroidism and approach to therapy. Neoreviews. 2020;21:e37-44.

6. Solutions for Newborn Screening. Perkin Elmer; 2020. https://new bornscreening.perkinelmer.com.

7. Benneyan JC, Lloyd RC, Plsek PE. Statistical process control as a tool for research and health improvement. Qual Saf Health Care. 2003;12:458-64. 
8. Provost LP, Murray S. The health care data guide: learning from data for improvement. Hoboken, NJ: Wiley; 2011.

9. Cheung YY, Jung B, Sohn JH, Ogrinc G. Quality initiatives: statistical control charts: simplifying the analysis of data for quality improvement. Radiographics. 2012;32:2113-26.
10. Cochran AL, Tarini BA, Kleyn M, Zayas-Caban G. Newborn screening collection and delivery processes in Michigan birthing hospitals: strategies to improve timeliness. Matern Child Health J. 2018;22:1436-43. 